\title{
Pensamento Computacional: Uma Proposta de Ensino com Estratégias Diversificadas para Crianças do Ensino Fundamental
}

\author{
Fernanda de Melo Reis, Fábio Cristiano Souza Oliveira, Danielle Juliana da Silva \\ Martins, Patrícia da Rocha Moreira
}

\author{
Instituto Federal de Educação, Ciência e Tecnologia do Sertão Pernambucano - \\ Campus Petrolina (IF SERTÃO PE - Campus Petrolina). \\ CEP 56.314-520 - Petrolina - PE - Brasil \\ fernanda.melreis@gmail.com, fabio.cristiano@ifsertao-pe.edu.br, \\ danielle.juliana.martins@gmail.com, patdarocha@gmail.com
}

\begin{abstract}
This article aims to present teaching and learning approaches, used with elementary school children to teach concepts of Computer Science and programming of computers in games, in order to work on computational thinking, evidencing in a learning that gives the students feeling. Thus, this proposal aims to report on the application of educational strategies such as Depluged Computing, Storytelling, Gamification and Significant Learning, with elementary students. We used a quantitative approach methodology, with information collected, in the field of action. For this, the students worked at different times both individually and in groups.
\end{abstract}

Resumo Este artigo visa apresentar abordagens de ensino e aprendizagem, utilizadas com crianças do ensino fundamental para o ensino de conceitos da Ciência da Computação e programação de computadores em jogos, a fim de trabalhar o pensamento computacional, evidenciando em uma aprendizagem que dê sentindo aos estudantes. Assim essa proposta tem como objetivo relatar a aplicação de estratégias educacionais como a Computação Desplugada, Storytelling, Gamificação e Aprendizagem Significativa, com alunos do ensino fundamental. Utilizou-se uma metodologia de abordagem quantitativa, com informações colhidas, no campo de ação. Para isso, os alunos trabalharam em momentos distintos tanto individualmente quanto em grupos.

\section{Introdução}

Desde as séries iniciais, as escolas estão introduzindo crianças e jovens para o ensino de conceitos de ciência da computação e programação de computadores, a fím de desenvolver o pensamento computacional dos estudantes. Inicialmente o ensino de conceitos e conteúdos relacionados a programação podem ser complexos, entre as principais dificuldades segundo Jenkins (2002, citado por Esteves et al., 2008) é o baixo nível de abstração, a falta de competências de resolução de problemas e a inadequação dos métodos pedagógicos aos estilos de aprendizagem dos alunos. Devido a essa 
problemática, busca-se estratégias que consigam maximizar o entendimento dos estudantes a fim de proporcionar-lhes o real sentido do que se está sendo apresentado, fazendo-os construir uma aprendizagem significativa.

O presente trabalho tem como objetivo relatar resultados acerca da experiência com estudantes do ensino fundamental, utilizando as metodologias de ensino como o Storytelling, que por sua vez significa aprender por intermédio de histórias, como também a Computação Desplugada, que trabalha conceitos de computação, sem o uso do computador, e a Gamificação, que proporciona elementos tradicionalmente encontrados nos games. Dessa forma, essa proposta defende a abordagem de metodologias de ensino diferenciadas, em busca de estimular habilidades do pensamento computacional nos estudantes.

As seguintes seções irão ser apresentadas da seguinte forma, na seção 2 serão abordados os trabalhos relacionados, onde são apresentados levantamentos sobre iniciativas relatando aplicações que incentivaram o uso da computação desplugada, storytelling, gamificação e aprendizagem significativa. A seção 3 apresentará as abordagens educacionais utilizadas para o ensino. Na seção 4 será descrita a metodologia, e estratégias utilizadas no trabalho. Na seção 5 serão relatados os resultados obtidos, e na seção 6 a conclusão e trabalhos futuros.

\section{Trabalhos Relacionados}

O trabalho de Pinho et al (2016), versa com a proposta deste trabalho, pois apresenta uma implementação junto com uma aplicação de um jogo educativo, utilizando uma metodologia que estimula habilidades específicas do pensamento computacional e estratégias de gamificação com computação desplugada, para despertar o interesse dos alunos. Outra experiência que colabora com essa proposta, é o trabalho de Gomes, Melo \& Tedesco (2016), que apresenta uma experiência de ensino de conceitos de programação para crianças utilizando jogos digitais, destacando sequência de instruções e repetição. Outro estudo descrito pelo trabalho de Oliveira (2012), é apresentado como uma proposta de ensino-aprendizagem para disciplinas de programação com recurso ao Storytelling e a robótica educacional, que estimula a criatividade e interesse dos estudantes, visando demonstrar a elevada taxa de insucesso na disciplina. Contudo, vale mencionar que o trabalho de Máverick et al. (2016) é muito relevante nesse contexto, pois é feita uma aplicação com alunos do ensino fundamental, onde é realizada uma análise, e revisão dos dados em relação a estratégias de gamificação.

As propostas e experiências ora descritas visam contribuir para a sustentação do trabalho, pois no decorrer da experiência utilizou a gamificação para compor estratégias de avaliação dos estudantes, como também a computação desplugada, juntamente com o pensamento computacional, utilizados para facilitar o ensino de conceitos complexos. Destacando também o ensino de programação de computadores, através da programação em blocos por meio de uma sequência de passos, condições e repetições de ações, além da storytelling para tematizar as aulas, proporcionando um enredo que envolvesse os conteúdos, e por fim, a aprendizagem significativa para valorizar o conhecimento prévio dos estudantes em relação aos conteúdos. 
VI Congresso Brasileiro de Informática na Educação (CBIE 2017)

Anais do XXIII Workshop de Informática na Escola (WIE 2017)

\section{Fundamentação Teórica}

Nesta seção serão pontuadas as estratégias e métodos de ensino utilizadas, que por sua vez são importantes para o entendimento do trabalho como um todo. Como já foi mencionado, os conceitos da ciência da computação podem se tornar muito complexos para iniciantes. Para Bell et al. (2011), sem o uso do computador e de forma descontraída, a Computação Desplugada pode ser empregada em uma sala de aula normal e fundamentos importantes da computação podem ser ensinados de forma fácil aos alunos. Com isso, conforme (Scaico et al. 2012; Souza et al. 2010), tais atividades desplugadas têm despertado o interesse de professores e pesquisadores, e tem sido empregada em diversos países ao redor do mundo.

Vale destacar também que a gamificação de acordo com (Werbach e Hunter, 2012), consiste em um método que faça a utilização de elementos dos games (mecânicas, estratégias, pensamentos) fora do contexto dos games. Kapp (2007) define Gamificação de forma semelhante, mas enfatiza o uso da mecânica, estética e raciocínio correntes nos jogos, como forma de engajar as pessoas, conduzi-las na solução de problemas e promover os processos de aprendizagem.

Nesse sentido, a experiência ora descrita utilizou a estratégia Storytelling com o propósito de contextualizar o conteúdo trabalhado em sala de aula. Desse modo, conforme (Brasil, 1998), temos a oportunidade de representar papéis e cenas do cotidiano, tomando posições e solucionando problemas de forma livre, sem a intervenção das pressões da realidade, podendo experimentar outras formas de ser e pensar. Dessa forma, é importante considerar a importância da aprendizagem significativa nesse contexto, pois como afirma Gowin (1981), a aprendizagem significativa é considerada um processo de reorganização ativa de uma rede de significados pré-existentes na estrutura cognitiva do indivíduo, vale afirmar também, que só há ensino quando há captação de significados.

\section{Metodologia}

Essa seção apresenta os métodos, estratégias e técnicas adotadas para a realização do curso de programação em jogos, realizado com treze alunos do $8^{\circ}$ ou $9^{\circ}$ ano do ensino fundamental. $\mathrm{O}$ curso teve duração de seis meses com carga horária de 30 horas, sua estrutura é fundamentada nas metodologias de Gamificação, Computação Desplugada, Storytelling e Aprendizagem Significativa. Atualmente encontra-se em andamento, as aulas ocorre duas vez na semana, com duração de 02 horas (duas) horas cada, e é dividido em duas fases, nas quais abordamos conceitos de ciência da computação; programação estruturada nas linguagem Pascal; programação em jogos com programação em blocos, além de plataformas de desenvolvimento em jogos $2 \mathrm{D}$ e 3D.

A primeira fase do curso descrita com resultados quantitativos nesse trabalho já foi finalizada, e tem por finalidade pontuar conceitos da ciência da computação, programação de computadores e em jogos 2D. Nesse sentido, no decorrer da prática, a gamificação foi empregada como método avaliativo, através de um sistema de ranking, 
VI Congresso Brasileiro de Informática na Educação (CBIE 2017)

Anais do XXIII Workshop de Informática na Escola (WIE 2017)

onde em cada aula, os alunos tinham uma missão que teria desafios referente ao conteúdo abordado em sala para ser solucionado, sendo que essas missões resolvidas lhes rendiam uma quantidade de pontos.

Vale mencionar, que as experiências que fizeram uso da computação desplugada neste trabalho envolveram missões que relacionassem conteúdos vistos na aula. Durante a execução das atividades buscou-se exemplificar as possíveis contribuições dos conteúdos apresentados, para então fazer com que os estudantes percebessem o seu valor e aplicabilidade. Por fim, o emprego do Storytelling nesse contexto possibilitou aos alunos mais chances de compreender situações possibilitando um enredo envolvendo o conteúdo proposto. Contudo, na seção 4.1 será apresentada a dinâmica das aulas, destacando o conteúdo trabalhado, além das missões propostas em cada encontro.

\subsection{Dinâmica das aulas}

A seguir irão ser mostrada a Tabela 1. onde serão apresentados os conteúdos trabalhados na primeira fase do curso.

Tabela 1. Conteúdos abordados na primeira fase do curso.

\begin{tabular}{|c|c|c|}
\hline Conteúdo da aula & Missão & O que foi trabalhado \\
\hline $\begin{array}{c}\text { Introdução a computação e } \\
\text { Algoritmos da vida real }\end{array}$ & Pegue o pombo & $\begin{array}{c}\text { Conceito de hardware e software; } \\
\text { O que é um algoritmo e tipos de } \\
\text { algoritmos. }\end{array}$ \\
\hline Representando a Informação & Enviando mensagens secretas ${ }^{1}$ & $\begin{array}{c}\text { Diferença de dado e informação; e } \\
\text { Representação de base binária. }\end{array}$ \\
\hline Paradigmas de Programação & Mini Gincana & $\begin{array}{c}\text { Revisão de conceitos iniciais da } \\
\text { computação; Representação de } \\
\text { base binária; Algoritmo; ideia de } \\
\text { estrutura sequencial em } \\
\text { programação. }\end{array}$ \\
\hline Trabalhando o Pensamento & Monstros no planeta Zurônio ${ }^{2}$ & $\begin{array}{c}\text { Apresentar o pensamento } \\
\text { computacional, como identificar } \\
\text { umproblema; dividir o problema } \\
\text { em partes menores; abstrair os } \\
\text { detalhes; organizar e testar } \\
\text { hipóteses. }\end{array}$ \\
\hline Operadores em Programação & Montando o Quebra-cabeça & $\begin{array}{c}\text { Operadores lógicos e relacionais } \\
\text { em programação. }\end{array}$ \\
\hline
\end{tabular}

${ }_{1}^{1}$ Disponível em: <http://csunplugged.org/wp-content/uploads/2015/01/unplugged-book-v1.pdf>. Acesso em: jun. 2017.

${ }^{2}$ Adaptado e disponível em: <https://code.org >. Acesso em: jun. 2017. 
VI Congresso Brasileiro de Informática na Educação (CBIE 2017)

Anais do XXIII Workshop de Informática na Escola (WIE 2017)

\begin{tabular}{|c|c|c|}
\hline $\begin{array}{c}\text { Mecânica de jogos digitais e } \\
\text { programação em jogos }\end{array}$ & $\begin{array}{c}\text { Laboratório infinito A Era do } \\
\text { Gelo }^{3}\end{array}$ & $\begin{array}{c}\text { Programação em blocos utilizando } \\
\text { estrutura sequencial, condicional, } \\
\text { repetição e operadores relacionais. }\end{array}$ \\
\hline
\end{tabular}

Irá ser apresentado as metodologias de ensino aplicadas nas aulas, e seus procedimentos, para exemplificar como foram empregadas.

Tabela 2. Missões trabalhadas em cada aula na primeira fase do curso.

\begin{tabular}{|c|c|c|}
\hline Missão & Metodologia(s) & Habilidades estimuladas \\
\hline Реgие о ротьо & $\begin{array}{l}\text { Storytelling: Ajudar o Mutley e o Esquadrão } \\
\text { Abutre a pegar o pombo e conseguir medalhas. } \\
\text { Gamificação: Sistema de pontuação e competição } \\
\text { estabelecido. } \\
\text { Computação Desplugada: Através de passos } \\
\text { montar um avião de papel } \\
\text { Aprendizagem Significativa: Empregar o pré } \\
\text { conhecimento em fazer um avião de papel, e dar um } \\
\text { novo sentido a partir de passos, como se estivesse } \\
\text { construindo um algoritmo em programação. }\end{array}$ & $\begin{array}{ll}\text { - } & \text { Concentração; } \\
\text { - } & \text { Raciocínio lógico e } \\
& \text { algorítmico; } \\
\text { - } & \text { Abstração; } \\
\text { - } & \text { Criatividade. }\end{array}$ \\
\hline $\begin{array}{c}\text { Enviando mensagens } \\
\text { secretas }\end{array}$ & $\begin{array}{l}\text { Storytelling: Ajudar o Perry a decifrar a mensagem } \\
\text { secreta, e resgatar Phineas e Ferb a escapar do Dr. } \\
\text { Doofenshmirtz. } \\
\text { Gamificação: Sistema de pontuação. } \\
\text { Computação Desplugada: Decifrar por números } \\
\text { binários no papel e em cartões. } \\
\text { Aprendizagem Significativa: Empregar o pré } \\
\text { conhecimento em representação de números } \\
\text { binários e dar um novo sentido a partir de decifrar a } \\
\text { mensagem, e construir uma mensagem para ajudar } \\
\text { no sucesso da missão. }\end{array}$ & $\begin{array}{ll}\text { - } & \text { Abstração; } \\
\text { - } & \text { Raciocínio lógico } \\
& \text { matemático; } \\
\text { - } & \text { Testar soluções; } \\
\text { - } & \text { Pensamento Crítico; } \\
\text { - } & \text { Comunicação; } \\
\text { - } & \text { Colaboração em } \\
& \text { equipe. }\end{array}$ \\
\hline & $\begin{array}{l}\text { Gamificação: Sistema de pontuação; Sistema de } \\
\text { regras; divisão por fases; cooperação em time. } \\
\text { Computação Desplugada: Passar por uma trilha } \\
\text { de fios; Calcular um arremesso de bambolês; } \\
\text { Ordenar passos para trocar uma lâmpada; } \\
\text { Responder perguntas e percorrer o Caminho da } \\
\text { Incógnita; Percorrer um caminho com um pezão de } \\
\text { papelão em pouco tempo, proporcionando uma } \\
\text { aprendizagem por meio de erros e tentativas; } \\
\text { Decifrar mensagem em representação de base } \\
\text { binária. } \\
\text { Aprendizagem Significativa: } \\
\mathbf{1}^{\mathbf{0}} \text { fase - Empregar o pré conhecimento em } \\
\text { atividades do dia-a-dia como trocar uma lâmpada, } \\
\text { e transformar em um algoritmo sequencial; }\end{array}$ & $\begin{array}{l}\text { - Raciocínio lógico e } \\
\text { algorítmico; } \\
\text { - } \text { Colaboração em } \\
\text { equipe; } \\
\text { - Calcular estratégia; } \\
\text { - Aprendizagem com } \\
\text { tentativas e erros; } \\
\text { - Comunicação; } \\
\text { - Coordenação } \\
\text { - } \text { Petora; } \\
\text { - Decomsamento crítico; } \\
\text { - }\end{array}$ \\
\hline
\end{tabular}

\footnotetext{
${ }^{3}$ Disponível em: <https://studio.code.org/s/iceage/stage/1/puzzle/1>. Acesso em: jun. 2017.
} 
VI Congresso Brasileiro de Informática na Educação (CBIE 2017)

Anais do XXIII Workshop de Informática na Escola (WIE 2017)

\begin{tabular}{|c|c|c|}
\hline Mini Gincana & $\begin{array}{l}\mathbf{2}^{\mathbf{0}} \text { fase - Utilizar um bambolê e calcular o seu } \\
\text { arremesso, em uma certa distância para alcançar o } \\
\text { colega de equipe; } \\
\mathbf{3}^{\mathbf{0}} \text { fase - Passar por uma trilha de fios e calcular } \\
\text { uma estratégia que use menos tempo e que evite } \\
\text { quebrar algum fio; } \\
\mathbf{4}^{\mathbf{0}} \text { fase - Usar o pré conhecimento em conceitos } \\
\text { iniciais da computação e representação da } \\
\text { informação para conseguir saltar sobre um caminho } \\
\text { até a linha de chegada; } \\
\mathbf{5}^{\mathbf{0}} \text { fase - Calçar um "pezão" de papelão, e percorrer } \\
\text { um caminho em menos tempo, que possibilite a } \\
\text { aprendizagem por meio de erros e tentativas; } \\
\mathbf{6}^{\mathbf{0}} \text { fase - Utilizar o pré conhecimento em } \\
\text { representação de base binária, para decifrar uma } \\
\text { mensagem. }\end{array}$ & $\begin{array}{ll} & \text { problema; } \\
\text { - Sistematização do } \\
\text { pensamento; } \\
\text { - Abstração. }\end{array}$ \\
\hline $\begin{array}{c}\text { Monstros no planeta } \\
\text { Zurônio }^{4}\end{array}$ & $\begin{array}{l}\text { Storytelling: Ajudar a identificar alguns monstros } \\
\text { no planeta Zurônio, desenhando seus retratos } \\
\text { falados em base de algumas descrições de } \\
\text { testemunhas oculares. } \\
\text { Gamificação: Sistema de pontuação, sistema de } \\
\text { regras e colaboração do time. } \\
\text { Computação Desplugada: Folha de papel e } \\
\text { canetas coloridas para os programadores relatar as } \\
\text { características dos monstros, para posteriormente os } \\
\text { decodificadores desenharem em base das instruções } \\
\text { os rostos dos monstros. } \\
\text { Aprendizagem Significativa: Usar o pré } \\
\text { conhecimento nas etapas que compõem o } \\
\text { pensamento computacional para resolver o } \\
\text { problema dos monstros no planeta Zurônio, como } \\
\text { abstrair os detalhes para identificar um padrão que } \\
\text { pareça válido na descrição dos monstros, dividindo } \\
\text { o problema em partes menores, além de organizar } \\
\text { cada um dos monstros na forma de listas de } \\
\text { instruções para desenhar e criar. }\end{array}$ & $\begin{array}{ll}\text { - } & \text { Abstração; } \\
\text { - } & \text { Raciocínio lógico } \\
& \text { algorítmico; } \\
\text { - } & \text { Criatividade; } \\
\text { - } & \text { Reconhecimento de } \\
& \text { padrões; } \\
\text { - } & \\
& \text { pecomposição de } \\
\text { - } & \text { Coordemas; } \\
& \text { motora; } \\
\text { - Sistematização do } \\
\text { pensamento; } \\
\text { Concentração. }\end{array}$ \\
\hline $\begin{array}{c}\text { Montando o } \\
\text { Quebra-cabeça }\end{array}$ & $\begin{array}{l}\text { Gamificação: Sistema de pontuação; e colaboração } \\
\text { em time. } \\
\text { Computação Desplugada: Peças que compõem } \\
\text { um quebra-cabeça de emborrachado, que contém } \\
\text { um código específico para cada equipe, onde cada } \\
\text { equipe deve conquistar uma peça do quebra-cabeça } \\
\text { que contém operadores lógicos, estrutura } \\
\text { sequencial, condicional e de repetição em } \\
\text { programação. } \\
\text { Aprendizagem significativa: Adivinhar o desenho, } \\
\text { ilustrado pelo integrante de time e conquistar uma } \\
\text { peça do quebra cabeça, por fim usar o pré }\end{array}$ & $\begin{array}{l}\text { - Raciocínio lógico, } \\
\text { matemático } \\
\text { algorítmico; } \\
\text { - Sistematização do } \\
\text { pensamento; } \\
\text { - Criatividade; } \\
\text { - Coordenação } \\
\text { motora; } \\
\text { - Testar soluções; }\end{array}$ \\
\hline
\end{tabular}

${ }^{4}$ Adaptado e disponível em: <https://code.org>. Acesso em: jun. 2017. 
VI Congresso Brasileiro de Informática na Educação (CBIE 2017)

Anais do XXIII Workshop de Informática na Escola (WIE 2017)

\begin{tabular}{|c|c|c|}
\hline & $\begin{array}{l}\text { conhecimento em estrutura sequencial, } \\
\text { condicional, repetição e operadores lógicos em } \\
\text { programação, para montar o quebra-cabeça do } \\
\text { código e explicá-lo corretamente. }\end{array}$ & $\begin{array}{l}\text { - Abstração; } \\
\text { - Comunicação. } \\
\text { - Colaboração em } \\
\text { equipe. }\end{array}$ \\
\hline $\begin{array}{l}\text { Laboratório Infinito } \\
\text { A Era do Gelo }\end{array}$ & $\begin{array}{l}\text { Storytelling: Ajudar os personagens Sid, Manny, } \\
\text { Diego, e Scrat, programando seus movimentos com } \\
\text { programação em jogos para realizar ações } \\
\text { específicas e criar um jogo em 2D. } \\
\text { Gamificação: Sistema de pontuação; pensamento } \\
\text { de jogo; e sistema de progresso em fases. } \\
\text { Aprendizagem Significativa: Usar o pré } \\
\text { conhecimento em programação como estrutura } \\
\text { sequencial, condicional e repetição, para programar } \\
\text { os personagens para executar movimentos } \\
\text { específicos criando um jogo em 2D. }\end{array}$ & $\begin{array}{ll}\text { - } & \text { Abstração; } \\
\text { - Raciocínio lógico e } & \text { algorítmico; } \\
\text { - } & \text { Pensamento de } \\
& \text { jogos; } \\
\text { - } & \text { Testar soluções; } \\
\text { - } & \text { Criatividade; } \\
\text { - } & \text { Inventividade. }\end{array}$ \\
\hline
\end{tabular}

\subsection{Sistema de Avaliação}

No final de cada aula, havia uma missão onde a avaliação dos alunos se deu exclusivamente por meio de um sistema gamificado, através de um sistema de ranqueamento onde os estudantes realizavam missões que valiam um quantitativo de pontos, estes conquistados pelos alunos foram lançados no site do projeto Academia HackTown ${ }^{5}$, onde cada aluno tem acesso a sua conta, e acompanha seu progresso individual, além do seu progresso em grupo.

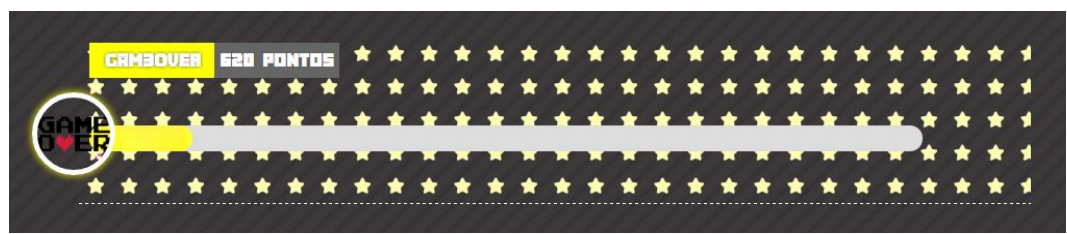

Figura 1. Ranking individual - site do projeto Academia HackTown.

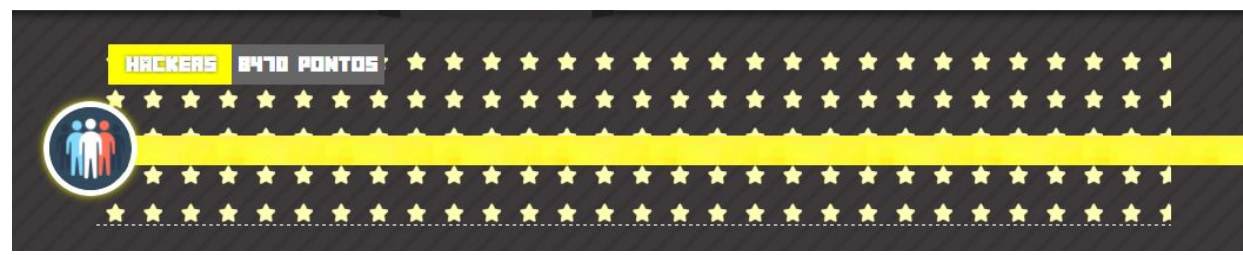

Figura 2. Ranking de grupo - site do projeto Academia HackTown.

\section{Análise dos Resultados}

Com relação aos dados quantitativos colhidos no campo de ação, buscou-se fazer uma análise dos resultados através de dados estatísticos extraídos através da aplicação de um questionário com duas questões, apresentada sob forma de itens da escala Likert de 7

\footnotetext{
${ }^{5}$ Disponível em: <http://hacktown.petrolina.ifsertao-pe.edu.br/ranking/>. Acesso em: mar. 2017.
} 
pontos, variando de 1 (Não classificado), 2 (Péssimo), 3 (Ruim), 4 (Razoável), 5 (Bom), 6 (Ótimo) e 7 (Excepcional), sendo respondido anonimamente por 13 (treze) alunos.

$\mathrm{Na}$ figura 3, apresenta-se as respostas para a pergunta "De 1 à 7, aponte seu nível de satisfação em realizar missões com competições:”. Neste quesito, apresenta-se uma razoável variação entre as medidas de tendência central (média, moda e mediana), e dispersão abaixo de $2 \%$, indicando uma unanimidade nas respostas dos estudantes, representada pelo índice do coeficiente de variação, correspondente a 1,3\%, o que significa que eles estão muito satisfeitos com as atividades e desafios propostos que possuem um perfil competitivo. A representação visual está no gráfico da Figura 3, indicando uma representatividade de nível superior estimada em 53,8\% dos alunos que participaram da pesquisa.

Tabela 3. Análise descritiva das respostas a satisfação dos estudantes sobre missões com competições.

\begin{tabular}{|c|c|c|c|c|c|}
\hline Tratamento & Média & Moda & Mediana & DP & CV\% \\
\hline 1 & 6,15 & 7 & 7 & 1,14 & $1,3 \%$ \\
\hline
\end{tabular}

Nota: $\mathrm{DP}=$ desvio padrão; $\mathrm{CV}=$ coeficiente de variação.

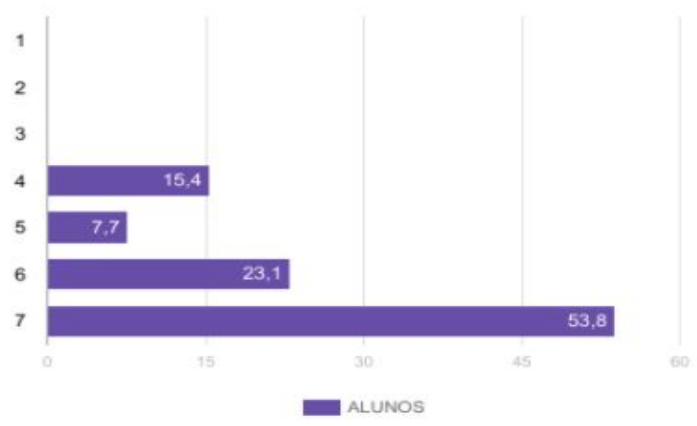

Figura 3. Gráfico representando a avaliação do nível de satisfação dos alunos em realizar missões com competições.

$\mathrm{Na}$ figura 4, apresenta-se as respostas para a questão sobre "O que você achou de ter aulas gamificadas com um sistema de pontuação e ranqueamento?”. Neste quesito, apresenta-se uma razoável variação entre as medidas de tendência central (média, moda e mediana), e dispersão abaixo de $20 \%$, caracterizando uma pequena variação nas respostas dos estudantes, indicando um número de mais da metade dos estudantes em concordância com a questão perguntada, representada pelo índice do coeficiente de variação, correspondente a 17,4\%, o que significa que estudantes estão muito satisfeitos em ser avaliados através de um sistema gamificado. A representação visual está no gráfico da Figura 4, indicando uma representatividade de nível superior estimada em $69,2 \%$ dos alunos que participaram da pesquisa. 
VI Congresso Brasileiro de Informática na Educação (CBIE 2017)

Anais do XXIII Workshop de Informática na Escola (WIE 2017)

Tabela 4. Análise descritiva das respostas sobre o sistema de pontuação e ranqueamento.

\begin{tabular}{|c|c|c|c|c|c|}
\hline Tratamento & Média & Moda & Mediana & DP & CV\% \\
\hline 2 & 6,46 & 7 & 7 & 1,12 & $17,4 \%$ \\
\hline
\end{tabular}

Nota: $\mathrm{DP}=$ desvio padrão; $\mathrm{CV}$ = coeficiente de variação.

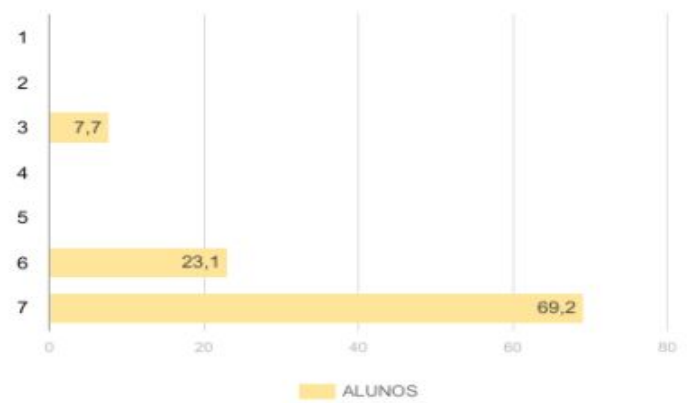

Figura 4. Gráfico representando o percentual das respostas dos alunos sobre ter aulas gamificadas com um sistema de pontuação e ranqueamento.

\section{Considerações Finais e Trabalhos Futuros}

Vale ressaltar, que as estratégias e métodos utilizados sempre objetivaram estimular os alunos durante as atividades (missões) realizadas em sala de aula, como também desenvolvendo, conceitos importantes como cooperação, criatividade e inventividade. Neste trabalho buscou-se demonstrar através de dados a utilização e eficácia das metodologias ora descritas para a aprendizagem dos estudantes, apresentando uma aceitação dos estudantes, pelas estratégias e sistema de pontuação proposto pela gamificação, relacionando-se diretamente com a motivação dos estudantes.

Dessa forma, vale destacar que as outras metodologias apresentaram uma aceitação superficial por parte dos estudantes, mas contudo, a gamificação fez um grande diferencial no processo de aprendizagem, motivando-os a conquistarem pontos e avançar no entendimento dos conteúdos abordados em sala de aula. No entanto, as lições aprendidas com a experiência foram de que metodologias que abrangeram as atividades não tradicionais contextualizadas no processo educacional, conseguem uma maior chance de envolver o estudante, possibilitando-o a potencializar habilidades específicas no fim desse processo, como capacidade de decompor e resolver problemas, raciocínio lógico, concentração e sistematização do pensamento, abstração do conteúdo, criatividade e inventividade, que vão além do seu uso na sala de aula.

Por fim, recomenda-se que trabalhos que utilizam alguma metodologia apresentada, e que desejam colaborar com o estímulo de habilidades relacionadas com o pensamento computacional, devem apresentar um olhar mais crítico sobre essas habilidades, correlacionando seus benefícios, buscando dados quantitativos que comprovem esse fato. Contudo, como perspectivas futuras de trabalho, busca-se apresentar um olhar mais crítico em relação as habilidades do pensamento computacional e demonstrar através de dados quantificáveis a validade das percepções 
VI Congresso Brasileiro de Informática na Educação (CBIE 2017)

Anais do XXIII Workshop de Informática na Escola (WIE 2017)

dos alunos, através do ensino, apontados no decorrer dessa experiência.

\section{Referências}

Almeida, M. E. B. \& Prado, M. E. B. B. (1999). Um retrato da informática em educação no Brasil. Disponível em: <http://www.proinfo.gov.br>. Acesso em: 18 de março de 2017.

Bell, T., Witten, I. H., and Fellows, M. (2011). Computer science unplugged. ensinando ciência da computação sem o uso do computador. Tradução por: Luciano Porto Barreto. Disponível em: <http://csunplugged.org/books>. Acesso: 18 de março de 2017.

Brasil, (1998). Referencial Curricular Nacional para Educação Infantil. Vol. 3. Brasília: MEC/SEF.

Oliveira, D. et al. (2012) Uma proposta de ensino-aprendizagem de programação utilizando robótica educativa e storytelling. In: II Congresso Internacional TIC de Educação, Lisboa. p. 10.

Dohme, V. (2000). Técnicas de contar histórias. 7. Ed. São Paulo: Informal.

Esteves, M., Fonseca, B., Morgado, L., Martins, P. (2008). Uso do Second Life em Comunidade de Prática de Programação. Prima.com, 6, 19-31.

Gomes, T; Melo, J; Tedesco, P. (2016). Jogos Digitais no Ensino de Conceitos de Programação para Crianças. In: Brazilian Symposium on Computers in Education (Simpósio Brasileiro de Informática na Educação-SBIE). p. 470.

Gowin, D. B. (1981). Educating. Ithaca, N.Y.: Cornell University Press. 210 p.

Jayasinghe, U.; Dharmaratne, A. (2013). Game based learning vs. gamification from the higher education students' perpective. Teaching, Assessment and Learning for Engineering (TALE). IEEE International Conference on, p. 683-688.

Kapp, K. M. (2007). Gadgets, games, and gizmos for learning: tools and techniques for transferring know-how from boomers to gamers. [S.1.]: John Wiley and Sons.

Máverick, D. et al. (2016). Análise, Revisão e Aplicação da Abordagem para Inclusão do Licenciado em Computação no Ensino Básico (ABILSEN). In: Anais do Workshop de Informática na Escola. P. 525.

Pinho, G. et al. (2016). Proposta de Jogo Digital para Dispositivos Móveis: Desenvolvendo Habilidades do Pensamento Computacional. In: Brazilian Symposium on Computers in Education (Simpósio Brasileiro de Informática na Educação-SBIE). p. 100.

Scaico, P.; Henrique, M.; Cunha, F. e Alencar, Y. (2012). "Um Relato de Experiências de Estagiários da Licenciatura em Computação com o Ensino de Computação para Crianças". Revista Renote: Novas Tecnologias na Educação, v. 10, n. 3.

Souza, R.; Barreto, L; Andrade, A.; e Abdalla, D.; (2010). "Ensinando e aprendendo conceitos sobre ciência da computação sem o uso do computador: Computação Unplugged!!!". Congresso Brasileiro de Informática na Educação, v. 1, n.1.

Werbach, K.; Hunter, D. (2012). For The Win: How Game Thinking Can Revolutionize Your Business. Filadélfia, Pensilvânia: Wharton Digital Press. 